ORIGINAL ARTICLE

Volume ry Issue 2

\title{
Comparison of Direct Staffing Costs of Advanced Practice Providers and Residents in a High-Acuity Area of a Community Emergency
}

\section{Department}

Kyle B. Smith, MD', Christopher Kiefer, MD $^{2}$, Erica Shaver, MD $^{3}$, Kimberly D.

Quedado, MS, MA, PhD4, Thomas Marshall, MD5, Jerome Kerwin, MBA, MHA6, and Christopher Goode, MD?

ABSTRACT

Resident physicians and advanced practice providers (APPs) both have roles in providing care within emergency departments (ED). While both bring unique skill sets and capabilities to the health care team, little is known about the comparative financial impact of APPs and residents in a community ED. The objective of this study was to compare direct staffing costs per relative value unit (RVU) generated of emergency medicine (EM) residents and APPs in a community ED setting.
Author affiliations are listed at the end of this article.

Corresponding Author: Kimberly Quedado, PhD Dept. of Emergency Medicine, West Virginia University kimberly.quedado@hsc.wvu.edu

\section{KEYWORDS}

RVU, APP, Resident, Direct Cost Analysis

\section{INTRODUCTION}

Resident physicians and advanced practice providers (APPs), a term inclusive of both physician assistants and nurse practitioners, have a role in the delivery of care within emergency departments (ED). The Accreditation Council for Graduate Medical Education (ACGME) placed restrictions on the total clinical hours that residents may work, initially in 2003 and then updated to be more stringent in 2011. Therefore, many academic departments have turned to APPs as a strategy to compensate for the decreased availability of residents for clinical work..$^{1,2}$ Across the United States, ED visits continue to increasefrom 123 million visits nationally in 2008 to 138 million visits in 2017. As a result, organizations in the community and academic settings are augmenting provider staffing through multiple avenues, including but not limited to, additional APP coverage, creation of new residency programs, and expansion of existing residency programs. ${ }^{3,4}$

Due to Centers for Medicare and Medicaid (CMS) regulations prohibiting hospitals from billing independently for resident services, all patients seen by a resident physician must also be seen by an attending physician. ${ }^{5}$ Depending upon medical staff bylaws at individual institutions, patients seen by APPs may or may not require evaluation by attending physicians, making APPs an attractive alternative to staffing with resident physicians. This creates the potential for increased department productivity by not taking the time to discuss every patient with an attending physician. Given increasing patient volumes and restrictions on resident work hours, APPs are now utilized in both high and low-acuity areas of EDs as a cost-effective way to augment the productivity of attending physicians. Prior literature has analyzed resident and APP productivity in terms of relative value units (RVU) per hour, RVUs per patient, and patients seen per hour in the academic setting and community affiliate settings. However, these prior studies have not included any discussion or analysis of the direct employment costs associated with both APPs and resident physicians. ${ }^{6,7}$

As they progress throughout training, resident physicians have increased RVU productivity with each additional post-graduate year. ${ }^{8}$ Resident physicians are less expensive in terms of base salary than APPs, but often require more time spent in discussion with the attending physician. The autonomy of a well- 


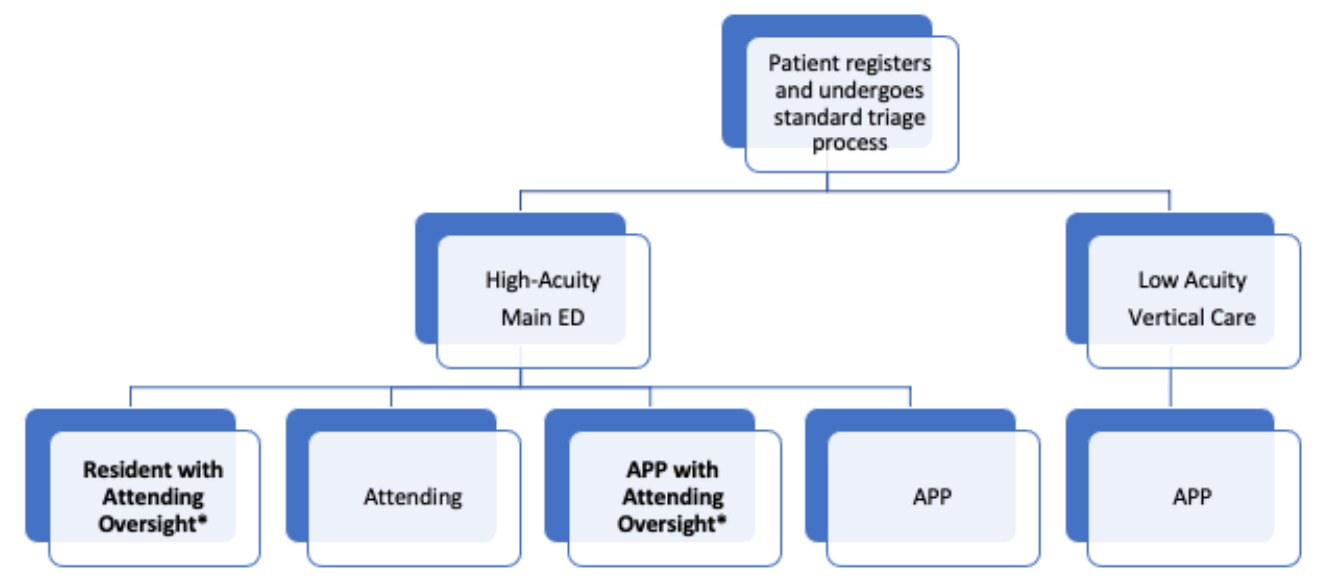

community ED serves as a secondary training site for emergency medicine residents and has a regular resident presence on site, with approximately 8,400 hours of emergency medicine resident coverage per year $(7,200$ hours from PGY2/3 residents and 1,200 hours from PGY1 residents).

FIGURE 1: This figure illustrates the triage process and attending staff patterns for patients presenting to the community ED in the study.

trained APP, when paired with an attending physician, leads to increased productivity, both in terms of RVUs and patients per hour, when compared to a resident/attending pairing. ${ }^{7}$ However, this increased productivity of the APP is associated with higher direct staffing costs when compared with resident physicians. It is unknown if the higher APP direct staffing costs are offset by increased productivity. The purpose of our study was to evaluate direct staffing costs per RVU generated by both APPs and EM resident physicians, per year of residency, in a high-acuity setting of a community ED where the attending physician evaluates and documents on each patient seen by both resident physicians and APPs.

Methods

This is a retrospective, observational analysis of the RVU productivity of resident physicians and APPs in a high-acuity area of a community ED. Located in rural Appalachia, the ED sees approximately 56,000 patients per year. Since the early 1990 s, the ED has been staffed by the American Board of Emergency Medicine board-certified/boardeligible attending physicians, and since 2005 has been affiliated with a university health system and department of emergency medicine that houses an emergency medicine residency program. The community ED currently annually funds 3.375 of the academic department's 30 total emergency medicine residency positions. As part of this affiliation, the
Staffing of patients in this community ED occurs through a vertical care/ split-flow model. Low-acuity patients are seen independently by APPs (Figure 1). The higher acuity main ED is staffed by attending physicians seeing patients independently, residents with attending oversight, APPs with attending oversight, and, on occasion, APPs evaluating patients independently. Although attending physicians are ultimately in a supervisory role over all APPs working in the ED, the attending physicians were only directly involved in evaluating select patients with the APPs, typically triggered by a request by the APP for supervision and guidance with particular patients.

Billing data was acquired from the electronic health record (ED Pulsecheck initially, followed by EPIC) and was analyzed to determine total RVUs generated by APPs and residents working with attending physicians during the three-year period (July 2015 to June 2018). Archived shift schedules were used to determine monthly hours worked by APPs and residents. Totals for hours worked and RVUs were used to calculate RVUs generated per hour for each group. Direct staffing costs were obtained for residents reviewing financial records for salary, fringe, and fixed expenses associated with residency program administration costs. APP costs included salary and fringe for the same three-year period. The aggregate of data costs per RVU generated was also determined for both groups. 
Total hours worked during the study period were between 7,710 and 9,803 hours per year for APPs and 8,056 to 8,408 hours per year for residents. PGY1 residents worked 1,208 to 1,216 hours per year while PGY2/3 residents worked from 6,840 to 7,200 hours with minor seasonal variations. RVU generation per year for APPs ranged from 22,057 to 29,883 , with an average RVU/hour generation of 2.584 to 3.048 , and a three-year average of $2.877 \mathrm{RVUs} /$ hour. PGY1, PGY2, and PGY3 residents produced 18,094, 28,868 , and 28,656 RVUs, respectively, during the study period, yielding resident physician RVU/hour calculations of 2.01, 3.00, and 3.49, respectively, for PGY1, PGY2, and PGY3. The mix of resident physicians studied produced an overall average of 3.06 RVUs/ hour (Table 1).

Staffing costs for APPs included a median hourly wage of $\$ 52.66$ per hour with a fringe rate of $22.7 \%$ combined, for a cost of $\$ 64.61$ per hour of APP coverage (Table 2). Average salaries during the three-year period of this study for the residents were $\$ 53,596$ (PGY1), $\$ 55,253$ (PGY2), and $\$ 56,844$ (PGY3), with a fringe rate of $28 \%$. Consistent with their contractual obligations, the community ED provided salary support for a total of 3.375 resident full-time equivalents (FTE's). The breakdown of community hospital FTE support by resident year was 0.375 for PGY1, 1.5 for PGY2, and 1.5 for PGY3.

site travel, continuing education, and oversight personnel costs, totaled $\$ 73,632.17$. Resident travel costs for reimbursement for the 70-mile round trip per shift to the community ED resulted in an estimate of $\$ 18,354$ per year. Continuing medical education (CME) funds were provided by the community hospital at a cost of $\$ 1,500$ per FTE, totaling $\$ 5,062.50$. In addition, the community hospital support also provides $11.25 \%$ of the salaries of related residency program personnel, including the residency program director, associate program director, and program manager. The combination of resident salary and administrative costs creates an overall resident cost to the community hospital of $\$ 318,358.25$ annually.

The hourly commitment per month to the community hospital was based on 10 PGY1 residents working approximately 120 clinical hours during their one-

The combination of average salaries with FTE commitment from the community hospital amounted to an average cost of $\$ 20,099$ for interns (0.375 FTE), \$82,880 for PGY2s (1.5 FTE), and $\$ 85,267$ for $P G Y 3 s$ (1.5 FTE) for the years 2015 through 2018. Overall annual administrative costs paid by the APP R1 R2 R3 Total Res

\begin{tabular}{cccccc}
\hline Salary & & $\$ 20,099$ & $\$ 82,880$ & $\$ 85,267$ & $\$ 188,245$ \\
\hline Hourly Rate & $\$ 52.66$ & $\$ 16.75$ & $\$ 23.02$ & $\$ 23.69$ & $\$ 22.41$ \\
Fringe & $\$ 11.95$ & $\$ 4.69$ & $\$ 6.45$ & $\$ 6.63$ & $\$ 6.27$ \\
Program Cost $/ \mathbf{H r}$ & & $\$ 8.77$ & $\$ 8.77$ & $\$ 8.77$ & $\$ 8.77$ \\
\hline Total Hourly Cost & $\mathbf{\$ 6 4 . 6 1}$ & $\mathbf{\$ 3 0 . 2 0}$ & $\mathbf{\$ 3 8 . 2 3}$ & $\mathbf{\$ 3 9 . 0 8}$ & $\mathbf{\$ 3 7 . 4 5}$
\end{tabular}
community hospital to support residents, including

TABLE 2: Calculation for Hourly Cost Based on Provider Type and Year of Training. 
month rotation at the community hospital, netting 1,200 hours of PGY1 coverage per year. Each of the twenty senior residents (PGY2 and PGY3) work 180 clinical hours during each of their two months at the community hospital, resulting in 7,200 hours of senior resident coverage annually. The combination of 8,400 resident work hours annually was consistent with the actual number of hours worked when compared to our data. The estimated hourly cost for resident physicians was calculated to be $\$ 30.20$ for PGY1s, $\$ 38.23$ for PGY2s, and $\$ 39.08$ for PGY3s utilizing the outlined salary and total administrative costs.

We then utilized the billing data for individual providers to determine RVU production in the highacuity/main ED when the patient was seen by either a resident/attending team or an APP/attending team during the same time period. The data were grouped by provider types (PGY1, PGY2, PGY3, and APPs) and were used to calculate a provider-specific average productivity per hour. The direct staffing cost per RVU generated was determined by dividing the direct staffing cost per hour by the number of RVUs generated per hour. On average, the direct staffing cost of an APP is approximately $\$ 22.46$ per RVU generated in a high-acuity environment where the patient is also seen by the attending. PGY1 direct staffing cost is $\$ 15.00$ per RVU generated, while PGY2s and PGY3s direct staffing costs are $\$ 12.75$ and $\$ 11.19$ per RVU generated, respectively. When a weighted average is calculated, the average direct staffing cost of a resident throughout three years of training is approximately $\$ 12.23$ per RVU generated, which is $45 \%$ less than their APP counterparts at $\$ 22.46$ per RVU.

\section{DISCUSSION}

Federal expansion of graduate medical education (GME) funding has failed to keep up with the needs of the emergency medicine workforce, resulting in an increase of privately funded residency programs. ${ }^{9}$ Additionally, ED visits continue to rise and many departments have turned to hiring APPs in both low and high-acuity environments to meet the demand. Emergency medicine residency positions have increased $36 \%$ over the past five years in that same effort. ${ }^{10,11}$ The number of corporate-associated residency training programs has increased due to low direct staffing costs, increased productivity of attending physicians when working with resident providers, and long-term recruitment potential. Previous research has demonstrated the positive financial impact of a new emergency residency program in a community ED setting, while other studies have demonstrated the higher RVU per patient productivity of residents compared to APPs in a high-acuity environment (Hamden). ${ }^{6,12}$ The annual operating cost a full-residency program during the previous study was $\$ 1,821,108$, while the expansion opportunity provided by the community site providing 3.375 resident FTEs had a cost of $\$ 318,358$ per year. In contrast to the Clinkscales experience of establishing a new residency program, the direct costs to the community hospital of resident salary and overhead are lower given our model as an expansion of an established residency program.

Our results provide evidence for the positive financial implications of residency program expansion into community EDs, given the higher RVU productivity of residents over APPs. We further defined these positive financial implications by evaluating, at the hourly level, the direct staffing costs per RVU generated. Our results show that APP direct staffing costs were $\$ 22.46 /$ RVU generated, while the average direct cost of a resident was $\$ 12.23 / \mathrm{RVU}$ generated. Given these findings, we conclude that the resident-attending team is twice as financially advantageous to the community hospital as the APP-attending team in a high-acuity area of community ED.

Previous studies show that APPs are more productive in terms of patients seen and RVUs generated in a fast-track setting. ${ }^{13}$ The same authors later found that PGY3 residents had higher RVU generation than APPs in a higher-acuity setting. ${ }^{6}$ Our data show that both PGY2s and PGY3s generate more RVUs per hour than APPs in the high-acuity setting when the attending is involved in every patient encounter. Although interns in this study generate fewer RVUs than all other groups and likely require more time and oversight, the time spent acclimating them to the community environment during their PGY1 year may foster this increased productivity seen in subsequent years of training. Furthermore, given the low cost of a resident physician, PGY1s continue to generate RVUs at a lower direct staffing cost per RVU generated than APPs. 
Our study took place in a community hospital that is part of the larger academic health system and is in close physical proximity ( 35 miles) to the primary training site. Through the funding of additional residency positions, the community hospital is able to maximize the benefits of having residents in training while not having to bear the full costs associated with operating a residency program. The positive financial impact is not the only potential benefit to the residency program and community hospital. Through continuous academic dialogue, assistance with procedures, and the co-management of high-acuity patients, the attending physicians may experience increased job satisfaction. For the resident physicians, the experience of working in a high-volume, high-acuity community environment unopposed by other residency and fellowship programs provides them with the opportunity to participate in all aspects of patient care, including advanced procedures and resuscitations that would often be shared with other specialties in the academic setting.

In this community hospital, the relationship between the residency program and the hospital spans over twenty years. In a state where many emergency departments utilize locums tenens physicians, our community hospital has not used locums coverage since the inception of the attending coverage contract in $2005 .{ }^{14}$ Of the 12 full-time physicians assigned to this practice, seven of them completed residencies in the associated emergency medicine residency program and rotated at the community hospital during their residency. The remainder of the attending physicians at this site desire a "hybrid" work environment that allows them to independently evaluate patients while having frequent bedside teaching opportunities with EM residents.

Operationally, the availability of EM residents allows the ED medical director to more efficiently staff the department by requiring lower attending physician and APP coverage, with the APPs being deployed more toward low-acuity patients and independent practice in fast-track setting where previous studies have demonstrated their increased productivity. ${ }^{6}$

Our study is not without limitations. It was conducted at a single site, currently in a steady-state period where resident physicians have been present for a prolonged period of time. It does not reflect operational issues that may arise in new programs or programs expanding to a new clinical site. This study does not address APP productivity in a model where they are practicing independently and does not represent their financial advantage when caring for low-acuity patients. Our study also did not take into account individual variation in resident performance or experience level of APPs. However, we feel that these variations are mitigated by regular fluctuation in staffing patterns whereby all providers, regardless of experience or post-graduate level, will work similar amounts of hours in a three-year study duration.

\section{CONCLUSION}

Financially, resident direct staffing cost per RVU generated is preferable to that of APPs in a highacuity community ED. The benefits to the community ED include lower overall staffing costs, improved recruitment, and continual flow of new knowledge and procedures into the institution. The additional benefits to the academic department include a distinct clinical learning environment, unopposed access to advanced procedures, and increased possibilities for scholarship-producing residency graduates with real-world community experience. Given the findings, community EDs should strongly consider partnering with accessible academic emergency medicine residency programs in order to financially support the expansion of the residency program, while synergistically enhancing operations of the community ED. 


\section{AUTHOR AFFILIATIONS}

1. Resident Physician, West Virginia University, Morgantown, West Virginia

2. Associate Professor, Associate Residency Program Director, Emergency Medicine, West Virginia University School of Medicine, Morgantown, West Virginia

3. Associate Professor, Vice Chair for Education and Training, Residency Program Director, Emergency Medicine, West Virginia University School of Medicine, Morgantown, West Virginia

4. Director of Research and Scholarship, Department of Emergency Medicine, West Virginia University School of Medicine, Morgantown, West Virginia

5. Vice Chair, Community Operations, Medical Director, Community Emergency Medicine, Emergency Medicine, West Virginia University School of Medicine, Morgantown, West Virginia

6. Administrator, Emergency Medicine, West Virginia University School of Medicine, Morgantown, West Virginia

7. Chair of Emergency Medicine, Emergency Medicine, West Virginia University School of Medicine, Morgantown, West Virginia

\section{REFERENCES}

1. Resident Duty Hours in the Learning and Working Environment: Comparison of 2003 and 2011 Standards online: Accreditation Council for Graduate Medical Education; [Available from: http://www.acgme.org/Portals/0/PDFs/dhComparisonTable2003v2011.pdf.

2. Freiburg C, James T, Ashikaga T, Moalem J, Cherr G. Strategies to accommodate resident workhour restrictions: impact on surgical education. Journal of surgical education. 2011;68(5):387-92.

3. National Hospital Ambulatory Medical Care Survey: 2008 Emergency Department Survey Tables. [June 1, 2020]. Available from: https:// www.cdc.gov/nchs/data/ahcd/nhamcs_ emergency/2008_ed_web_tables.pdf.

4. National Hospital Ambulatory Medical Care Survey: 2017 Emergency Department Survey Tables. [June 1, 2020]. Available from: https:// www.cdc.gov/nchs/data/nhamcs/web_ tables/2017_ed_web_tables-508.pdf.
5. Center for Medicare Services "Medicare Claims Processing Manual--Chapter 12 Physician/ Nonphysician practitioners online [Available from: https://www.cms.gov/Regulations-andGuidance/Guidance/Manuals/Downloads/ clm104c12.pdf.

6. Hamden K, Jeanmonod D, Gualtieri D, Jeanmonod R. Comparison of resident and mid-level provider productivity in a high-acuity emergency department setting. Emergency Medicine Journal. 2014;31(3):216-9.

7. McDonnell WM, Carpenter P, Jacobsen K, Kadish HA. Relative productivity of nurse practitioner and resident physician care models in the pediatric emergency department. Pediatric emergency care. 2015;31(2):101-6.

8. Brennan DF, Silvestri S, Sun JY, Papa L. Progression of emergency medicine resident productivity. Academic Emergency Medicine. 2007;14(9):790-4.

9. Gisondi M. How Competitive was Emergency Medicine in the 2018 Match? The answer might surprise you.: Academic Life in Emergency Medicine; 2018 [Dec 11, 2019]. Available from: https://www.aliem.com/match-2018-trends/.

10. Press Release: Thousands Of Resident Applicants Celebrate NRMP Match Results. 2019. [June 1, 2020] Available from: https://www.nrmp.org/ one-nine-press-release-thousands-residentphysician-applicants-celebrate-nrmp-matchresults/

11. Haas MR, Hopson LR, Zink BJ. Too Big Too Fast? Potential Implications of the Rapid Increase in Emergency Medicine Residency Positions. AEM Education and Training. 2020;4:S13-S21.

12. Clinkscales JD, Fesmire FM, Hennings JR, Severance HW, Seaberg DC, Patil N. The effect of emergency medicine residents on clinical efficiency and staffing requirements. Academic Emergency Medicine. 2016;23(1):78-82.

13. Jeanmonod R, DelCollo J, Jeanmonod D, Dombchewsky O, Reiter M. Comparison of resident and mid-level provider productivity and patient satisfaction in an emergency department fast track. Emergency Medicine Journal. 2013;30(1):e12-e.

14. Bennett P, Tadros A, Davis SM, Hobbs GR. The 2004 West Virginia emergency medicine workforce. The West Virginia medical journal. 2007;103(5):10-3. 